\title{
Digital Video Applications in Radiologic Education: Theory, Technique, and Applications
}

\author{
Joseph G. Hennessey, Elliot K. Fishman, and Derek R. Ney
}

\begin{abstract}
Computer-assisted instruction (CAI) has great potential in medical education. The recent explosion of multimedia platforms provides an environment for the seemless integration of text, images, and sound into a single program. This article discusses the role of digital video in the current educational environment as well as its future potential. An indepth review of the technical decisions of this new technology is also presented.
\end{abstract}

Copyright 1994 by W.B. Saunders Company

$\mathbf{D}^{2}$ URING THE PAST few years numerous articles have appeared expounding the virtues of computer-assisted instruction (CAI) in radiology. ${ }^{1-4}$ The advantages of CAI are far more than merely an alternative means of disseminating information. Compared with standard educational formats, the user of CAI can learn material faster with increased retention. ${ }^{5}$ One of the driving forces behind this educational revolution has been the development of relatively low-cost personal computers (PCs) that provide the user with a "multimedia environment." This environment usually refers to computer programs that allow the seamless integration of text, pictures, and sound into a single composite program. Recent advances in technology, specifically the introduction of digital video, promise to take the educational experience one step further. Digital video is the digital representation synchronized play-back of video and audio information on a computer. This article discusses digital video, its applications within the current educational environment, and its future potential. We will also provide an in-depth review of the technical decisions and compromises of this new and exciting technology.

\section{MATERIALS AND METHODS}

\section{Hardware and Software}

Hardware. The hardware required for creation and display of digital video consists of a computer (keyboard, monitor, hard drive, memory, central processing unit (CPU), a video cassette recorder (VCR), a sound digitizer, and a video digitizer board for the computer. For display, only the computer is required. In our laboratory, we used a Macintosh IIci computer (Apple Computers, Cupertino, CA) with 8 megabytes of random access memory, and a large (600 Mbyte) external hard-disk drive. The VCR was a VHS HQ model, the sound digitizer used was the MacRecorder (Farallon Computing, Emeryville, CA), and the video digitizer was a Video Spigot from SuperMac (SuperMac Technology, Mountain View, CA) along with a 24-bit video board from SuperMac for display.

Software. The software included the video sequencing program Premiere (Adobe Systems Inc, Mountain View, CA). Premiere is an excellent program for creating QuickTime (Apple Computer's form of digital video) videos, mirroring many of the features that would be found in professional analog equipment, such as many different kinds of wipes and fades, overlays, merging of multiple audio tracks, etc. The video and audio digitization software was ScreenPlay from SuperMac. ScreenPlay is the digitization program that is included with the Video Spigot and captures video and sound with minimal editing capabilities.

\section{Technical Considerations}

Overview. Digital video is the synchronized display of digitally sampled audio and visual signals. Digital video has many advantages; quality is not lost when duplicating or editing, and indexing is possible.

Digital video is usually accomplished as follows. First the subject material (eg, an imaging procedure) is captured on video tape using a normal video camera. Once the scene is recorded on video tape, it is necessary to transfer it to the computer. This involves sampling the analog signal to convert it to digital format and

From the Russell H. Morgan Department of Radiology and Radiological Science, at the Johns Hopkins Medical Institutions, Baltimore, $M D$.

Address reprint requests to Elliot K. Fishman, MD, Department of Radiology, Johns Hopkins Hospital, 601 N Caroline St, Baltimore, MD 21287.

Copyright 1994 by W.B. Saunders Company

0897-1889/94/0702-0009\$3.0010 
store it on the computer. This is done with a video and audio digitizer.

Depending on the computational and data rate limitations, some form of data compression may be required. Once the audio and video signals have been stored, they may be played back and edited as desired using appropriate software. Digital video playback software synchronizes the sampled audio and visual signals and presents them to the user.

Certain decision affecting digital video must be made at the point of converting between analog and digital format. For the audio portion of the program, choices that come into play include stereo versus monophonic sound; 16-bit versus 8 -bit sample size; and sampling rate. For video, the choices include color versus gray scale, bits per sample, frame rate, and frame size.

At the point of saving a file, certain choices must also be made such as what the final distribution method will be and what type of data compression will be required. The choices include lossy versus lossless data compression, compact disk read-only memory (CD-ROM) versus hard drive playback, and required hardware capability. The guiding concept behind these choices is that of data rate, which is the amount of information that can be transferred per time period. In the computer world, data rate is usually expressed in units of bits, bytes, kilobytes, or megabytes per second. Data rate is always limited by any bottlenecks in a system, eg, a traffic jam will most likely occur if even a short section of a four-lane highway is forced to only one lane by an automobile accident; the bottleneck in this case is the one open lane of traffic. Common bottlenecks for digital video are CD-ROM transfer rate, computer processing power, and graphics display speed.

Audio. The choice between monophonic and stereo sound involves the choice between one and two channels of sound, respectively. Music will sound better with two channels, whereas voice usually only requires one channel, cutting the size of the final sound in half. Sample size is the number of discrete steps in the amplitude of the sound. Excellent results can be attained with 16-bits per sample because this allows 65,536 different amplitudes (the parameter used for compact disc (CD) music recording). Under- standable voice can be attained with 8-bit sound containing 256 different amplitudes and only needing half the storage space. The final parameter is the sampling rate. The Shannon-Whitaker Sampling Theorem states that the highest frequency that can be accurately reproduced is half the sampling frequency. Thus, a CD-ROM with a sampling rate of $44.2 \mathrm{kHz}$ can accurately reproduce $22.1-\mathrm{kHz}$ sounds, which are beyond the upper range of most people's hearing ability. Music should be sampled at 22 to $44 \mathrm{kHz}$ (the higher the better) giving corresponding maximum frequencies of 11 to $22 \mathrm{kHz}$. Voice is still understandable with sampling rates of 5 to $11 \mathrm{kHz}$. The advantage of using $5.5 \mathrm{kHz}$ versus $44.2 \mathrm{kHz}$ sampling rate is that $5.5 \mathrm{kHz}$ only requires $1 / 8$ the storage of $44.2 \mathrm{kHz}$ for the same duration of sound. Depending on the choices above, storage requirements for the audio portion of digital video can vary by a factor of 10 or more. We generally use $11-\mathrm{kHz}$ monophonic sound for both voice and music; this is an acceptable trade-off for us because music is not as crucial to our applications and the frequency is commonly accepted by Macintosh editing software. A possible alternate to voice is the use of text narration as subtitles to the video, and QuickTime provides such an option for a substantial savings over voice on both data rate and storage size.

Visual. The use of color versus gray scale images is an important decision. Color images require three times more storage space then gray scale images because color must store a red, green, and blue channel whereas there is just one channel for gray scale. Additionally, gray scale is the obvious choice when the original source of data is in gray scale (eg, a series of computed tomographic [CT] scans).

Bits per sample (bits per pixel) is another important parameter. Eight bits per pixel gives 256 different levels of illumination, and is usually sufficient for displaying gray scale image. (Although not commonly available, more than 8 bits would be required to show the full information content of many medical images such as CT or magnetic resonance images.) For color, this corresponds to 24 bits ( 8 bits for each of the red, green, and blue channels) for each pixel. This gives $16,777,216$ possible colors with smooth transitions, but requires three times the storage 
of the equivalent gray-scale image. Some storage can be saved by using only 5 bits per channel per pixel for a total of 32,768 colors an image. This produces reasonable results using only 15 bits of storage per pixel but creates some banding artifacts in areas of slowly varying color.

Frame rate is another important factor. The continuity of motion in video is actually an illusion. The eye is tricked into seeing continuous motion whereas in reality there is only a sequence of still images. If the human mind and eye could react fast enough, we would see a fast slide presentation instead of motion. To produce the illusion of motion, a certain number of frames per second are required, otherwise the video appears jumpy. The number of frames per second needed for smooth motion varies to some extent from individual to individual, but is around $15 \mathrm{frame} / \mathrm{s}$. Movies use $24 \mathrm{frames} / \mathrm{s}$ and television uses 30 frames/s (interleaved so that half of a frame is updated every 60th of a second) to provide a smoother illusion. The eye can synthesize motion at less than 15 frames/s but the jumpiness gets progressively worse with lower frame rates, and rates less than $10 \mathrm{frames} / \mathrm{s}$ are not usable (except in a voice narration of a still scene, in which low frame rates or even stills would be useful). Thus, this criterion of 15 frames/s can be used to judge whether one has reached an acceptable frame rate for digital video (although even at $30 \mathrm{frames} / \mathrm{s}$ some flicker might still be noticeable and distracting) while requiring less storage than higher frame rates.

Frame size is a final important issue in the digitization of visual information. The visual aspect of digital video commonly consists of rectangular grid of samples known as pixels or pels. Common digital video standards are $640 \times$ $480,320 \times 240,240 \times 180$, and $160 \times 120$ pixels. These resolutions tend to maintain the approximate 4 to 3, width-to-height ratio found in the broadcast television signal. Reducing storage size can result in big savings of storage space. For example, reducing the frame from $640 \times$ 480 to $160 \times 120$ pixels reduces storage requirements by a factor of 16 (at the cost of losing small objects and detail in the original image).

Depending on the various choices made in digitizing, the visual and audial aspects of digital video storage requirements can vary by a factor of 100 or more. If low-quality digital video is acceptible, data compression may not be required. However for better-quality video, data compression is needed.

There are many issues involved when dealing with video data compression. The first issue is lossy versus lossless compression. Lossy data compression is a process in which an image that has been compressed and then uncompressed is different from the original. This means that the new image is not exactly like the old image, often having subtle (and sometimes not so subtle) artifacts. Lossless compression techniques retain all information from the original image, and therefore, are more suited for use in diagnostic applications. Lossless techniques will usually give compression ratios between 1.5:1 and $4: 1$. Lossy techniques can compress, on average, anywhere from $1.5: 1$ to $300: 1$, depending on the technique involved, the acceptable loss level (ie, how much of a decrease in image quality one is willing to accept because at high compression, the loss in quality can be quite noticeable), and CPU processing constraints. The use of lossy data compression is currently necessary because, even for gray-scale data (240-pixels width $\times 180$-pixels height at 15 frames/s), a compression rate of $4.2: 1$ is necessary for playback from standard CD-ROM players. Digital video can be compressed by spatial and (unlike still images) temporal techniques. Spatial data compression involves compression of similarities in single frames of data, whereas temporal data compression involves determination of similarities among linear sequences of frames. For example, temporal compression of a fixed view of a city street would optimally store only one copy of a parked car in the foreground because it does not change from frame to frame, whereas spatial compression would shrink the space needed to store the car because it could be described as a polygonal object of a certain color.

Macintosh implementation. Our digital video system is implemented with the Macintosh computer system (Apple Computers, Cupertino CA). There were certain design considerations involving audio and visual digitization as well as the compression method and the distribution method.

Data-rate limitations determined the various 
options for digital video. Full-screen video with CD-quality sound at television frame rates is the goal, but the data rate required is not presently attainable. Most CD-ROM's have a maximum input/output (I/O) data rate of approximately $150 \mathrm{kB}$ per second $(1 \mathrm{kB}=1,024$ bytes $)$. Playing full $\mathrm{CD}$-quality sound $(44.2-\mathrm{kHz} 16$-bit stereo sound) takes up this full data rate, leaving no room for video. Additionally, because of seek times and interleaving of audio, the maximum throughput of the CD-ROM for digital video is closer to $120 \mathrm{kB} / \mathrm{s}^{6}$ than the $150-\mathrm{kB} / \mathrm{s}$ maximum rate.

An additional audio constraint is that the Macintosh computers we used cannot play 44$\mathrm{kHz}$ 16-bit sound (CD quality) without additional hardware. They can only handle, at best, 8-bit sound at a $22-\mathrm{kHz}$ sampling rate ${ }^{6}$ (8-bit sound has only 256 possible amplitude values versus 16-bit sound with 65,536 possible amplitude values giving smoother variation). The two practical choices for audio use in QuickTime are $22-\mathrm{kHz} 8$-bit, and $11-\mathrm{kHz} 8$-bit monophonic sound. These two choices require $1 / O$ data rates of $22 \mathrm{kB} / \mathrm{s}$ and $11 \mathrm{kB} / \mathrm{s}$, respectively, leaving 98 $\mathrm{kB} / \mathrm{s}$ and $109 \mathrm{kB} / \mathrm{s}$, respectively, for video. A 22-kHz sampling rate should be used for recording music (because of the presence of relatively high frequencies in music); however, the 11 $\mathrm{KHz}$ sampling rate is sufficient for voice recording.

We chose a frame rate of 15 frames/s because it produced acceptable smoothness of motion at half the data-rate requirements of 30 frames/s.

A single frame of video uncompressed at $240 \times 180$ pixels would require approximately $127 \mathrm{kB}$ for 24-bit color or approximately $42 \mathrm{kB}$ for gray scale. Because of the large amount of data needed for color images, we choose to use 16-bit color requiring only $84 \mathrm{kB}$ (instead of 127 $\mathrm{kB}$ ). This is usually satisfactory for images that do not contain many continuous tones.

The question of frame size is another important issue. Using 336-pixel width $\times$ 483-pixel height requires $317 \mathrm{kB}$ for a 16-bit color image and requires a data rate of approximately 9.3 Mbytes/s for 30 frames/s, which would require a 64:1 compression ratio to play from CDROM. (This compression level is unobtainable on current Macintosh Computers without the use of additional hardware, such as a digital signal processor or compression chips.) By using a frame size of 240-pixel width $\times 180$-pixel height, only one quarter of the data is required, and therefore, data compression schemes giving a 17:1 compression ratio are sufficient.

On the Macintosh, there are five compression methods available. The first compression method is the Joint Photographic Expert Group (JPEG), named after the group that developed the standard. JPEG gives both the highest quality and greatest compression rates of the lossy compression methods available on the Macintosh, but there are several problems. First, JPEG is only spatial compression and does not involve temporal compression. The second problem with JPEG is that the Macintosh does not have sufficient processing power to decompress JPEG at acceptable frame rates for 240-pixel width $\times 180$-pixel height images. It can function at acceptable frame rates for images one quarter this size, but this is too small to be useful. The second compression method on the Macintosh is a form of run length encoding (RLE), which, according to Apple is the "Animation" method. This method applies RLE in both spatial and temporal dimensions. RLE involves replacing a sequence of similar data values with one data value and a count of the number of times it appears. The drawback of the Animation method is that it is highly sensitive to noise and therefore cannot provide good compression ratios for video with its moderate noise levels. However, for illustrations or drawings that are smooth and computer generated, very high compression rates can be achieved using RLE.

Another compression method available on the Macintosh is the Graphic compression method. The Graphic compression method is designed to dither 24-bit images down to 8-bit images using an error-diffusion dither method and then applying a lossy compression algorithm to them. The Graphics compression does not achieve a high enough compression ratio on video to be used with CD-ROM technology. Two other compression methods available on the Macintosh are the Video and Compact Video compression methods. These compression methods, which are lossy, use both temporal and spatial compression and achieve high enough compression ratios to playback from CD-ROM with moderate picture quality. The 
Video method will result in a compression ratio of approximately 10:1 with acceptable image quality. The Compact Video method is recommended whenever video is used as a source. It achieves compression ratios two or three times greater than attainable by the Video method at the cost of approximately 10 times greater compression time. ${ }^{7,8}$

Program distribution. Program distribution is another practical issue to be considered. With digital video movies running about 10 Mbytes/ min, even after compression (one of our current teaching programs on three-dimensional (3D) imaging requires well over 200 Mbytes of video), CD-ROM is the only cost-effective method of distribution because it can store about an hour of video. Floppy disks lack the storage capacity to store more than a few seconds of video each. Digital audio tape (DAT) could be used to distribute QuickTime movies but it lacks the capacity for nonsequential access-The DAT must be copied to a very large local hard drive. Magneto-optical cartridges are effective, but they require expensive drives and are not cost effective. Therefore, CD-ROM is the distribution method of choice. CD-ROM's greatest problem is that its maximum data-transfer rate of $150 \mathrm{kB} / \mathrm{s}$ was not designed to handle the $\mathrm{I} / \mathrm{O}$ data-rate requirements of video. This can be solved in two ways. First the entire CD-ROM can be copied to a large capacity hard drive. This decreases access time while increasing $\mathrm{I} / \mathrm{O}$ data rate to more than $1 \mathrm{Mbyte} / \mathrm{s}$ on most Macintosh II machines. This results in an approximate seven-fold increase in the amount of data that can be transferred per second; making larger, faster, higher-quality digital video possible. The second method of solving the memory transfer rate of CD-ROM is that there are now a number of CD-ROM players available that have two times the data-transfer rates (allowing $300 \mathrm{kB} / \mathrm{s}$ ) and even newer systems with four times the transfer rates (allowing an $\mathrm{I} / \mathrm{O}$ data rate of $600 \mathrm{kB} / \mathrm{s}$ ). This twofold to fourfold increase in speed would make larger, faster, higher-quality digital video possible.

Analog video disks are an alternate form of distributing video. Their advantages are that they are cheaper than a computer and store more images at even higher resolution than CD-ROM. (Although a CD-ROM could be used to store fewer still images at greater resolutions than the analog video disk, such stills are not useful for direct video playback.)

One of the greatest advantages of digital video over analog video disks is that one server can hold movies to be distributed over many networked computers. With video disks, each separate machine must have its own video-disk player and television monitor as well as a separate copy of the video disk itself. The cost of this is prohibitive, especially for large sites. Conversely, digital video can often take advantage of additional networking hardware and servers, thus making it more feasible to implement on a large scale.

\section{Radiologic Applications}

The initial radiologic applications of $\mathrm{CAI}$ combined the use of text with static images. With the increased sophistication of computer programs developed for multimedia, the combination of text, images, and questions became more seamless and the popularity of medical education programs grew. Recent articles in radiologic literature ${ }^{9}$ as well as exhibits at major meetings, including those of the Radiological Society of North America (as in the InfoRad section) and the American Roentgen Ray Society have shown the value and potential of computer-assisted education. With the introduction of digital video on the Macintosh computer, the potential for further development using clinical material now seems even more evident. QuickTime now allows the user to import video from any source, whether a $3 / 4$-inch tape, VHS tape, or $8-\mathrm{mm}$ media. Currently, projects that illustrate the potential advantage of video importation into the computer include a tutorial on 3D imaging, a radiologist's guide to laparoscopic cholecystectomy, and a tutorial on rheumatologic evaluation of the hand.

In the program on 3D imaging, we discuss clinical applications of $3 \mathrm{D}$ and allow the user to view actual video loops of the images. This allows the user to see examples of $3 \mathrm{D}$ clinical cases. In the section on laparoscopic cholecystectomy, the user can view a detailed analysis of the individual steps of an actual laparoscopic cholecystectomy. Finally, video images of an actual physical examination of the hand and 
wrist is presented in the rheumatologic program.

\section{CONCLUSION}

Digital video provides the opportunity to expand the capabilities of CAI. Although only in its infancy, the techniques are already pushing the hardware to its limits. The recurrent theme of $\mathrm{I} / \mathrm{O}$ data rate is seen to be the guiding principle in determining the limits of digital video. All aspects of the computer must be capable of handling the $\mathrm{I} / \mathrm{O}$ data rate-the necessary prerequisite for the use of digital video. Although the use of digital video is only beginning, it can be used effectively in applications today; and, in the future, it promises to enhance the nature of computerized radiologic education.

\section{REFERENCES}

1. Sinha S, Sinha U, Kangarloo H, et al: A PACS-based interactive teaching module for radiologic sciences. AJR Am J Roentgenol 159:199-205, 1992

2. Goldberg HI, Fell S, Joseph MH, et al: A computerassisted, interactive radiology learning program. Invest Radiol 25:947-951, 1990

3. Stein LD, Snydr-Michal J, Greenes RA: Realistic viewing and manipulation of radiographic images on a personal computer-A user interface for educational applications. J Digit Imaging 4:169-176, 1991

4. Tessler FN: Computer applications in radiologic education: A challenge for the 90's. AJR Am J Roentgenol $152: 1169-1172,1989$
5. Hennessey JG, Fishman EK, Kuhlman JE, et al: Computer-based learning in radiology: A hypermedia application in CT. AJR Am J Roentgenol 155:1317-1320, 1990

6. Apple Computer Inc: The Apple CD-ROM Handbook. Reading, MA, Addison-Wesley, 1992

7. Gonzalez RC, Woods RE: Sampling, in: Digital Image Processing. Reading, MA, Addison-Wesley, 111-119, 1992

8. Utz P: How your camcorder works. Camcorder Magazine 8:28-30,148, 1992

9. Rabushka LS, Fishman EK, Goldman SM, et al: Development of a computer-assisted instructional tool for evaluation and treatment of renal masses an experiment in hypermedia. Invest Radiol 28:169-174, 1993 